\title{
The diameters of guinea pig auditory nerve fibres: Distribution and correlation with spontaneous rate ${ }^{2}$
}

\author{
Otto Gleich * and Shirley Wilson ${ }^{1}$ \\ The Auditory Laboratory, Department of Physiology, The University of Western Australia, Nedlands, W.A. 6009, Australia ${ }^{c}$ Australian Neuromuscular \\ Research Institute, QE11 Medical Centre, Nedlands, Western Australia 6009, Australia
}

(Received 24 March 1993; Revision received 21 July 1993; Accepted 25 July 1993)

\begin{abstract}
In the mammalian auditory nerve physiological recordings revealed that the spontaneous discharge rate of single auditory fibres correlates with the diversity of input-output functions which may be important for intensity discrimination (e.g., Sachs and Abbas, 1974, Liberman, 1978; Winter et al., 1990). In this study we determined if the spontaneous discharge rate of auditory nerve fibres in the guinea pig is correlated with an anatomical feature, namely the diameter of the respective fibres. The diameter of myelinated (Type I) guinea pig auditory nerve fibres was measured after staining with different techniques. Measurements were made on semithin sections using a video image analysis system. The diameters of fibres stained with toluidine blue from the portion of the auditory nerve containing fibres from the basal turn of the cochlea were found to have a normal distribution. Fibres were also labelled with horseradish peroxidase by bulk injection into the spiral ganglion. It was found that the presence of horseradish peroxidase within the fibres reduced the measured diameter in comparison to adjacent unlabelled fibres. A number of fibres were physiologically characterized with respect to spontaneous discharge rate and subsequently intracellularly labelled with horseradish peroxidase. Fibre diameter of a selected sample of intracellularly labelled fibres was measured over a distance of $800 \mu \mathrm{m}$ within the internal auditory meatus. At the positions nearest to the spiral ganglion fibres possessing low spontaneous rates were found to have smaller diameters than high spontaneous rate fibres. No difference in fibre diameter was found for the positions near the cochlear nucleus.
\end{abstract}

Hearing; Cochlea; Horseradish peroxidase; Single cell labelling; Video image analysis

\section{Introduction}

The mammalian auditory nerve is composed of fibres which differ in their patterns of hair cell innervation, morphological characteristics and responsc properties to sound. The majority of these fibres are afferent fibres, $90-95 \%$ of which innervate the inner hair cells of the cochlea (Spoendlin, 1972). This group of afferent fibres have been named Type I fibres (Spoendlin, 1971) and are myelinated, spontaneously active and responsive to sound. The remaining $5-10 \%$ of afferent fibres, referred to as Type II fibres, innervate outer hair cells. These are small diameter fibres which are usually unmyelinated (Spoendlin, 1979; Liberman, 1982; Kiang et al., 1982). So far only one Type II cell has been recorded from and this fibre did not respond to sound (Robertson, 1984).

Type I fibres have been further categorized on the

\footnotetext{
* Corresponding author. Present address: HNO-Klinikum Universität Regensburg, Postfach, 93042 Regensburg, FRG.

2 Present address: Australian Neuromuscular Research Institute, QE11 Medical Centre, Nedlands, W.A. 6009, Australia.

2 Some preliminary results of this study have been presented at the 1991 ARO Midwinter Research Meeting
}

basis of spontaneous discharge rate (SR) and threshold at characteristic frequency, the sound frequency to which the fibre is most sensitive (Liberman, 1978; Liberman, 1982; Robertson, 1984; Rhode and Smith, 1985, Winter et al., 1990). Liberman (1978) proposed three categories for Type I fibres in the cat on these bases: high SR for fibres with SRs greater than 18 spikes per second, medium SR for fibres with SRs between 0.5 and 18 spikes per second and low SR for fibres with SRs less than 0.5 spikes per second. In a number of species high SR fibres have the lowest thresholds, medium SR fibres have intermediate thresholds, and low SR fibres have the highest thresholds (Liberman 1978; Schmiedt, 1989; Winter et al., 1990).

Other response properties have since becn shown to vary with SR. In both cats and guinea pigs the fibres within the three SR categories differ in the shape of their rate-intensity functions (Liberman, 1988, Sachs et al., 1989; Winter et al., 1990; Yates et al., 1990), and in the range of sound intensities they detect (dynamic range). In guinea pig high SR fibres have a dynamic range of 20 to $30 \mathrm{~dB}$ while low SR fibres have a dynamic range in excess of $60 \mathrm{~dB}$ (Winter et al., 1990).

Morphologically, Type I fibre diameters differ but they are unimodally distributed (Gacek and Ras- 
mussen, 1961; Arnesen and Osen 1978; Friede, 1984; Spoendlin and Schrott, 1989). After intracellular injection of horseradish peroxidase (HRP) into fibres of known SR Liberman and Oliver (1984) showed in cat that differences in fibre diameter were correlated with SR. Low SR fibres had on average smaller diameters and high SR fibres had the largest diameters. This difference was greatest in the dendritic portion peripheral to the spiral ganglion, but was still evident in the segment between the spiral ganglion and the cochlear nucleus.

There are other morphological differences in fibre diameter that are not associated with SR. In the cat, squirrel monkey and mouse, fibres emanating from the basal turn of the cochlea are presumably smaller than apical turn fibres (Alving and Cowan, 1971; Arnesen and Osen, 1978; Anniko and Arnesen, 1988). However, the diameters of basal and apical fibres are not significantly different in humans (Spoendlin and Schrott, 1989) but in the guinea pig apical fibres were reportedly smaller than basal fibres (Friede, 1984). Furthermore fibre diameter also varies along the VIIIth nerve trunk. In cat, guinea pig and human the portion distal to the spiral ganglion has fibres with smaller diameters than the portion between the spiral ganglion and the cochlear nucleus (Liberman and Oliver, 1984; Brown, 1987; Spoendlin and Schrott, 1989).

To date there has been no detailed investigation of the correlation between axonal morphology and physiological properties in the guinea pig. The purpose of this study was to determine the distribution of fibre diameters and to describe the relationship between axonal diameter and SR in this species.

\section{Methods}

\section{Animal preparation}

A total of 26 guinea pigs (weighing 260-620 g) were used in this study. The animals received approximately $0.3 \mathrm{mg} / \mathrm{kg}$ atropine sulphate subcutaneously prior to anaesthesia. Ten minutes later they were given 25 $\mathrm{mg} / \mathrm{kg}$ Nembutal (pentobarbitone sodium) intraperitoneally followed by Hypnorm (1.77 mg fluanisone and $0.035 \mathrm{mg}$ fentanyl per $\mathrm{kg}$ ). This regimen induced surgical anaesthesia for 3-4 h, as checked by the hindlimb withdrawal reflex, but did not interfere with normal respiration in healthy animals. All incisions were infiltrated with $2 \%$ Lignocaine solution. Surgical preparation and recording generally lasted $3-4 \mathrm{~h}$, until the animals showed first signs of recovery from the anaesthesia. In a small number of experiments an additional dose of $4.5-5.3 \mathrm{mg} / \mathrm{kg}$ Nembutal and Hypnorm (0.6$0.9 \mathrm{mg}$ fluanisonum and $0.012-0.017 \mathrm{mg}$ fentanylum per $\mathrm{kg}$ ) was administered in order to continue neural recordings.

\section{Recording and HRP injection}

The animal was placed in a sound-attenuating chamber on an automatically-controlled heating pad. The head was firmly mounted between two ear bars which also served as the housing for the closed speaker system. An incision behind the ear and careful dissection of the muscle layer allowed access to the bulla. Compound action potential thresholds were monitored using a silver wire placed near the round window. Opening of the bulla exposed the basal turn of the cochlea. To gain access to auditory nerve fibres an opening was made in the bony wall of scala tympani, using techniques described previously (Alder and Johnstone, 1978; Winter et al., 1990).

For bulk injections of HRP into the spiral ganglion of two animals a piece of bone overlying the ganglion was removed (Robertson and Manley, 1974). About 0.1-0.2 $\mu \mathrm{l} 30 \%$ aqueous HRP-solution was pressure injected into the ganglion using a glass micropipette with a tip diameter of approximately $20 \mu \mathrm{m}$.

For recording and staining single auditory nerve fibcrs within the modiolus (21 animals), a small holc was made in the bone overlying the modiolus (Alder and Johnstone, 1978). Electrodes were bevelled and back-filled with 10\% HRP-solution (Robertson, 1984), and advanced through the small hole into the auditory nerve. Upon encountering a single fibre, spontaneous rate was determined from a single or several 5 s samples. Subsequently the frequency versus threshold curve was measured, generally in $1 \mathrm{kHz}$ steps using an automated threshold tracking programme (Winter et al., 1990). In this study we were interested in a possible correlation between SR and the fibre diameter. Thus, in order to minimize possible effects of the CF (characteristic frequency; frequency where the unit is most sensitive) that might influence fibre diameter (Alving and Cowan, 1971; Arnesen and Osen, 1978; Friede, 1984; Anniko and Arnesen, 1988) we biased the CFs of the cells in this study towards high frequencies (15$24 \mathrm{kHz}$ ). When the electrode was intracellular, as indicated by a negative $10-30 \mathrm{mV}$ step, HRP was iontophoretically applied from the electrode by depolarising current pulses of $2-10 \mathrm{nA}(50 \mathrm{~ms}, 10 / \mathrm{s})$ for $10 \mathrm{~min}$. The electrode was left in place for $10 \mathrm{~min}$ after the injection, and then advanced further in order to impale other axons.

After the HRP injections the hole in the bony wall of scala tympani was covered with a small piece of gelatine film. Lignocaine solution $(0.5-0.8 \mathrm{ml})$ was applied around the muscle layer and skin incisions. These were closed by 3-4 stitches and the animals were allowed to recover.

\section{Histological processing}

Approximately $24 \mathrm{~h}$ after the last HRP injection the animals were killed with an overdose of Nembutal. 
They were perfused through the heart with $80 \mathrm{ml}$ of heparinized $0.9 \%$ saline. This was followed by $400 \mathrm{ml}$ $2.5 \%$ glutaraldehyde in $0.1 \mathrm{M}$ phosphate buffer of $\mathrm{pH}$ 7.4 over a period of approximately $30 \mathrm{~min}$ and then 400 $\mathrm{ml}$ of $0.1 \mathrm{M}$ phosphate buffer.

The temporal bone, with the complete auditory nerve and the cochlear nucleus attached, was removed from the skull. Most of the bulla, the tympanic membrane and the middle ear ossicles were removed to expose the cochlea. Care was taken not to stress or break the auditory nerve. Further dissection was either performed immediately after this step, or after storage of the specimen in $0.1 \mathrm{M}$ phosphate buffer overnight at $4^{\circ} \mathrm{C}$. In order to expose the auditory nerve, excess bone was removed using a dental drill with small diamond burrs. The final result of such a dissection is shown in Fig. 1A.

Specimens were then decalcified for 3-5 days in 0.1 $M$ EDTA solution in $0.1 \mathrm{M}$ phosphate buffer. During this period specimens were gently agitated and kept at $4^{\circ} \mathrm{C}$. After decalcification the auditory nerve was severed as close as possible to the cochlea.
The auditory nerve including the cochlear nucleus was kept in $30 \%$ sucrose in $0.1 \mathrm{M}$ phosphate buffer for $12-24 \mathrm{~h}$ at $4^{\circ} \mathrm{C}$ on a rotator until the tissue sank to the bottom of the vial and was then agitated for $1-12 \mathrm{~h}$ in albumen gelatine solution (20\% egg albumen, $0.5 \%$ gelatine, $30 \%$ sucrose in $0.1 \mathrm{M}$ phosphate buffer). The tissue was embedded in the albumen gelatine mixture which was hardened by the addition of a few drops of glutaraldehyde solution. After 20-30 min blocks were trimmed to an orientation which enabled cross-sectioning through the auditory nerve.

Serial $80 \mu \mathrm{m}$ cross-sections of the auditory nerve were cut on a freezing microtome. They were subjected to a cobalt intensified diaminobenzidine reaction for demonstration of HRP labelling as described by Liberman and Oliver (1984).

After reaction, sections were osmicated in $1 \% \mathrm{OsO}_{4}$ in phosphate buffer for $15 \mathrm{~min}$, washed thoroughly in 6 changes of phosphate buffer, dehydrated in a graded series of alcohol, and via propylene oxide infiltrated with araldite. The sections were placed in serial order with a small drop of araldite between two thick trans-
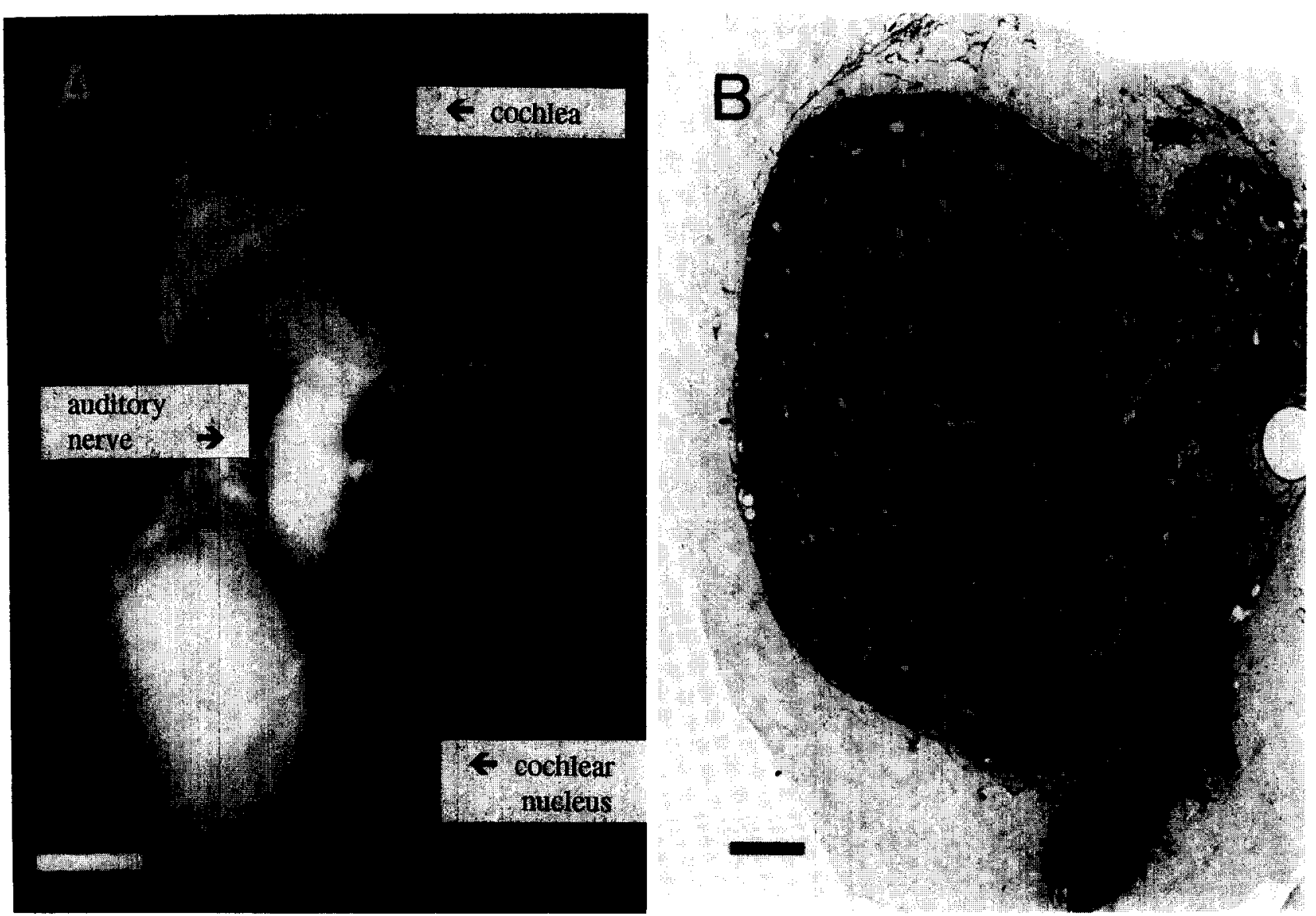

Fig. 1. (A) The dissected cochlea, auditory nerve and cochlear nucleus of a guinea pig. Calibration scale bar $1 \mathrm{~mm}$. (B) Photomicrograph of a cross-section of the auditory nerve $360 \mu \mathrm{m}$ central from Scarpa's ganglion with toluidine blue stained fibres. Fibres in the portion marked with the asterisk and bounded by the dotted line were analysed. Calibration scale bar $100 \mu \mathrm{m}$. 
parent polyethylene sheets. These 'sandwiches' were polymerized for $24-36 \mathrm{~h}$ at $65^{\circ} \mathrm{C}$ and stored for later processing.

After removal of one of the polyethylene sheets it was possible to select individual sections which were removed from the sheet and glued onto a flat araldite block. Subsequently they were resectioned at 0.5 or 1 $\mu \mathrm{m}$ and sections from within a $15 \mu \mathrm{m}$ segment of the nerve were mounted on separate clean microscope slides. Some slides were stained for $5 \mathrm{~min}$ with a hot aqueous solution of $1 \%$ toluidine blue, $1 \%$ azure II and $1 \%$ borax (as shown in Fig. 1B). Finally they were
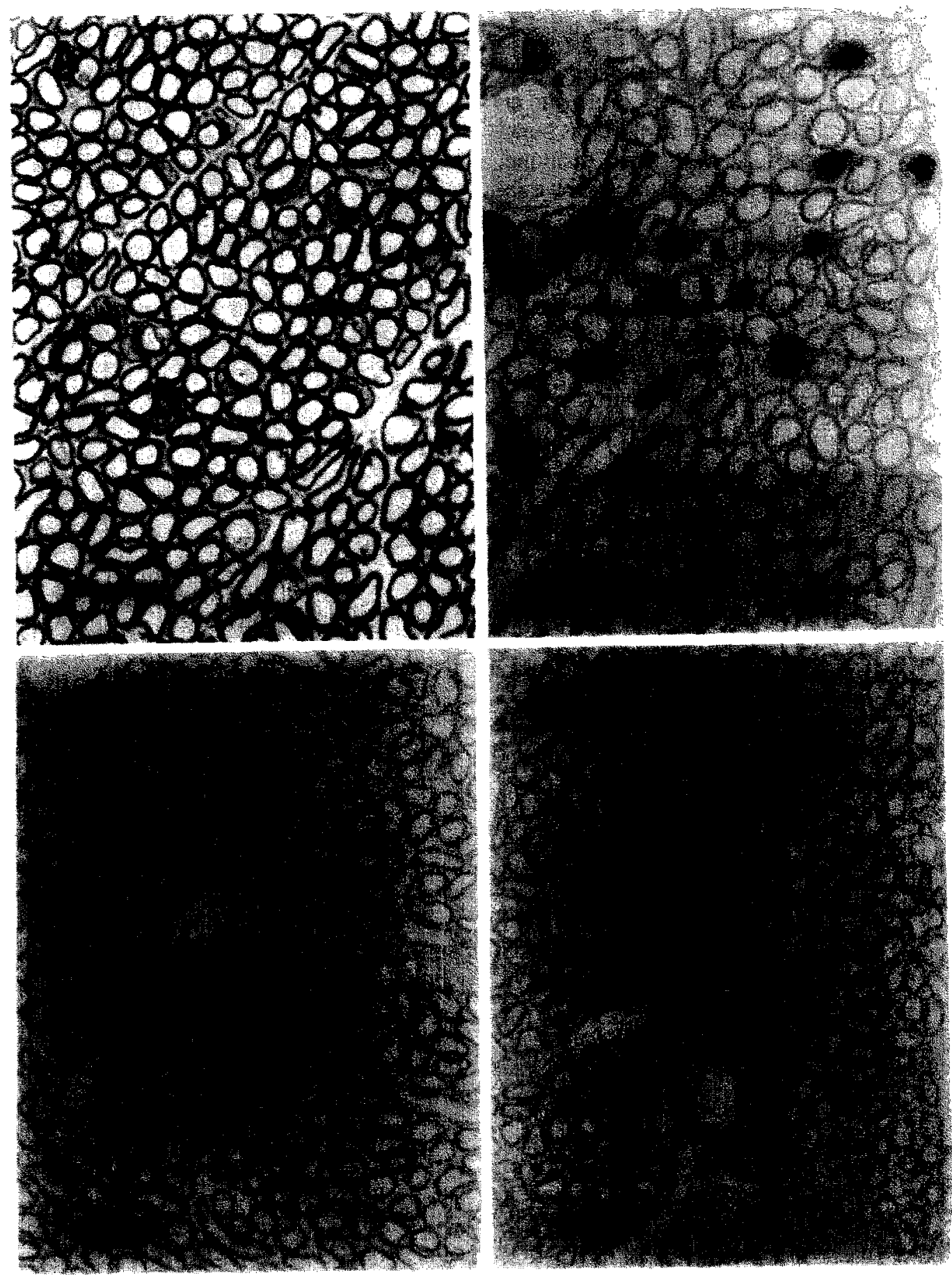

Fig. 2. Photomicrographs of cross-sections of auditory nerve fibres. (A), Toluidine blue stained fibres; (B), fibres stained with HRP after bulk injection into the spiral ganglion; (C) and (D), a low SR fibre (C) and a high SR fure (D) labelled by intracellutar wijetion of HRP; section thickness A-D 0.5 or 1 m. Calibration scale bar $10 \mu \mathrm{m}$. 
embedded in Permount and coverslipped. Fig. 2 shows examples of material stained with toluidine blue (Fig. 2A), material labelled by bulk HRP injection into the spiral ganglion (Fig. 2B) and material labelled by intracellular injection of HRP (Figs. 2C and 2D).

\section{Morphological analysis}

Images of auditory nerve fibres for analysis were obtained using a light microscope (Olympus Vanox-T) with a $100 \times$ oil immersion lens and a high resolution video camera (Mintron, CCD camera). For single fibre

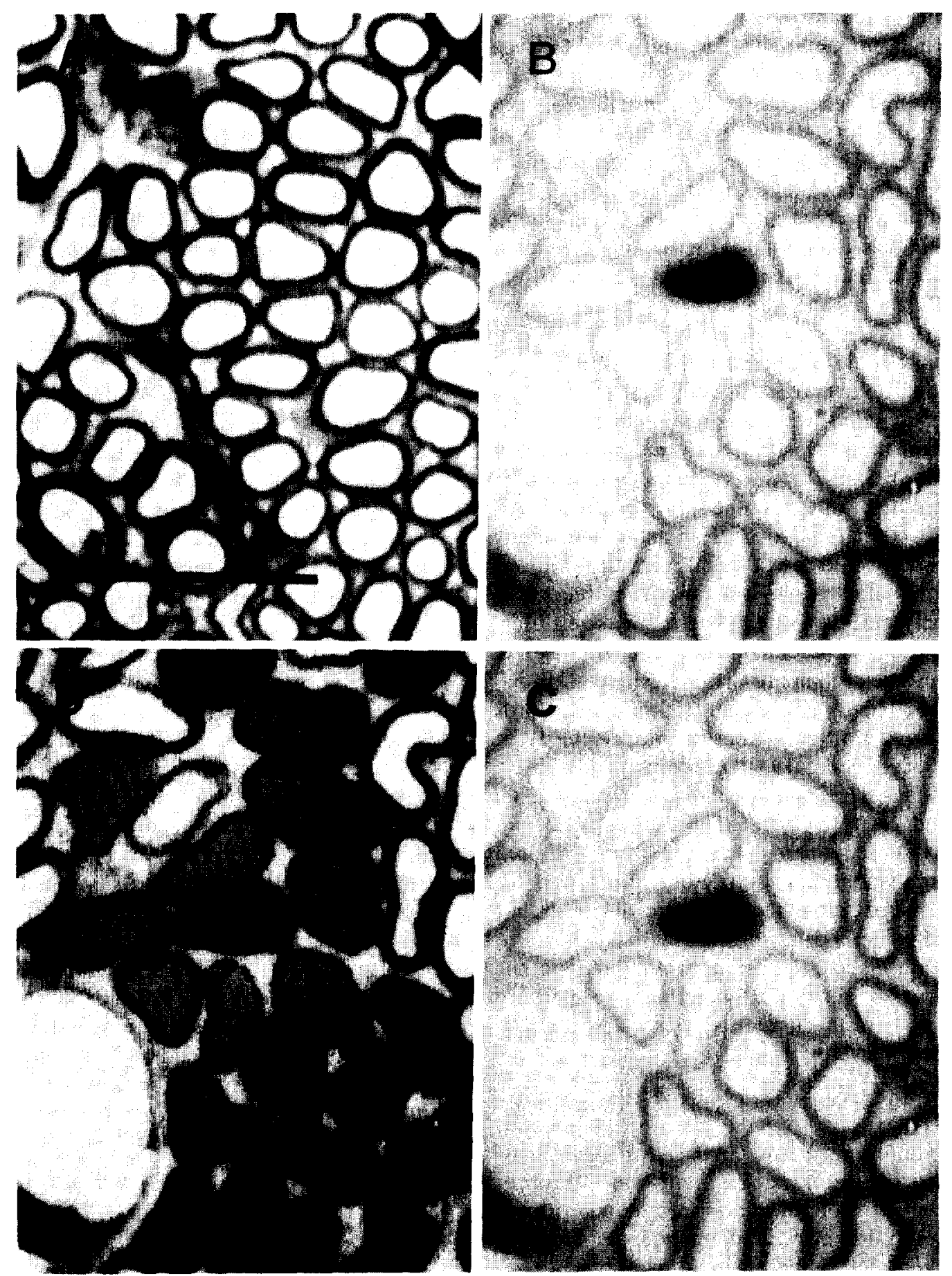

Fig. 3. Photographs of video images of auditory nerve fibres taken from the monitor screen. (A), Toluidine blue stained fibres showing differentiation between myelin and axoplasm; (B-D), video image of a section containing one fibre labelled by intracellular injection of HRP. This image is from the section shown in Fig. 2D. (B), Image before pixel selection, (C), with axoplasm selected and marked, (D), with axoplasm of some neighbouring unlabelled fibres selected and marked. Calibration scale bar A-D $10 \mu \mathrm{m}$. 
studies six images were obtained for each labelled fibre, each image being from a separate section within a $15 \mu \mathrm{m}$ segment of the nerve. The video images were stored for subsequent analysis using an image analysis system (MD-20 Image Analysis System, L.R. Jarvis, Flinders Imaging). With the configuration we used the resolution was $0.078 \mu \mathrm{m}$ per pixel, equivalent to a final $9000 \times$ magnification (calibrated using a Zeiss graticule). With this system structures could be selected for subsequent measurements by selecting pixels. Each pixel was assigned a grey value between 0 (black) and 63 (white). In our specimens the myelin and axoplasm had different grey levels allowing specific selection of axoplasm (Fig. 3). The selection was accomplished by selecting all pixels above or below the grey level of the myelin. For toluidine blue and lightly osmicated material the myelin was darker than the axoplasm, and so grey levels above the chosen threshold were selected, and for HRP material the axoplasm was darker so grey levels below threshold were selected. The selected pixels were displayed in a different colour. Inappropriatcly sclected structures (c.g. extracellular space; blood vessels) were excluded from the analysis by the operator. The system then measured area and perimeter of all contiguous patches that were made up by pixels within the predetermined range of grey levels. $\mathrm{Al}$ though the cross-sectional area of an axon determined by this method would be a good measure for the characterization of physiological properties (e.g. conduction velocity) and would emphasize any size differences among axons we decided to determine an axonal diameter in order to compare our material with previousely published data (Gacec and Rasmussen, 1961; Alving and Cowan, 1971, Arnesen and Osen, 1978; Liberman, 1982; Friede, 1984; Liberman and Oliver, 1984; Brown, 1987; Anniko and Arnesen, 1988; Spoendlin and Schrott, 1989). Thus, internal axonal diameter was subsequently calculated from the area measurements assuming circularity of the profiles. Although some axonal cross sections were obviously not true circles, this method has previously been shown to have the greatest accuracy when compared to other methods (Karnes et al., 1977). Shape factor, a measure for the deviation from circularity, was calculated according to the following formula:

shape factor $=10-\left((40 \times\right.$ area $\times \mathrm{pi}) /\left(\right.$ perimeter $\left.\left.^{2}\right)\right)$

A value of 0 represents a true circle (having the smallest possible perimeter for a given area) while 10 represents the largest possible deviation from circularity.

Statistics of differences in diameters among fibre populations were determined using a two tailed $t$-test.

The care and use of the animals reported on in this study were according to Australian laws.

\section{Results}

Distribution of fibre diameter

Fig. 2A shows the typical appearance of auditory nerve fibres from the basal half of the cochlea in 0.5 $\mu \mathrm{m}$ cross sections of non-HRP injected material stained with toluidine blue. The portion of the nerve analysed is indicated in Fig. 1B. This portion of the nerve was selected because it contained predominantly fibres from the basal turn of the cochlea which are tuned to high frequencies. Thus the diameters of these fibres can be directly compared with those of the intracellularlylabelled high-CF fibres and those labelled after HRP bulk injection into the spiral ganglion of the basal turn. The diameters of the axons in the non-HRP injected specimen were analysed at two levels between Scarpa's ganglion and the cochlear nucleus. One was at the most peripheral end of Scarpa's ganglion and the other $360 \mu \mathrm{m}$ more central towards the cochlear nucleus. In Fig. 4A the distribution of fibre diameter of auditory nerve fibres $360 \mu \mathrm{m}$ central from Scarpa's ganglion is shown. For this position the internal diameters ranged from approximately 1 to $5 \mu \mathrm{m}$ with a mean of $2.65 \mu \mathrm{m}$ (S.E.M. $=0.004 \mu \mathrm{m}, N=14173$ ) and were normally distributed. The same analysis was performed for a position at the peripheral edge of Scarpa's ganglion. In order to reduce the amount of data only every second field of view (equivalent to the black or white fields on a chess board) was analysed. The distribution at the more peripheral position was normal with a mean diameter of $2.70 \mu \mathrm{m}$ (S.E.M. $=0.01 \mu \mathrm{m}, N=2668$, range $1-4 \mu \mathrm{m}$ ).

Fibre diameter in horseradish peroxidase stained material

The axon diameter of cells filled with HRP by bulk injection into the spiral ganglion of the basal turn were analysed using the same methods. To determine possible effects of the different processing procedures diameters were also obtained for the unlabelled axon profiles immediately adjacent to the HRP labelled cells. The sections used were $1 \mu \mathrm{m}$ cross sections at the level $360 \mu \mathrm{m}$ central to Scarpa's ganglion (Fig. 2B). Fig. $4 \mathrm{~B}$ shows the diameters of fibres labelled with HRP by bulk injection. The distribution of fibres was normal for both the HRP-labelled and the neighbouring unlabelled fibres. The mean diameter of HRP fibres was smaller $(2.70 \mu \mathrm{m}$, S.E.M. $=0.03 \mu \mathrm{m}, N=428)$ than that for unlabelled fibres $(2.97 \mu \mathrm{m}$, S.E.M. $=0.01 \mu \mathrm{m}$, $N=1839$ ). These differences between the mean diameters of HRP-filled and unlabelled axons were significant $(P<0.01 ; t$-test).

The results shown in Fig. 4B were pooled from two animals, however, the variation between animals was minimal (Table I). For the HRP labelled fibres the mcan diameter from animal 90a was $2.63 \mu \mathrm{m}$ (S.E.M $=0.04 \mu \mathrm{m}, N=142$ ) and for animal 91a it was 2.73 

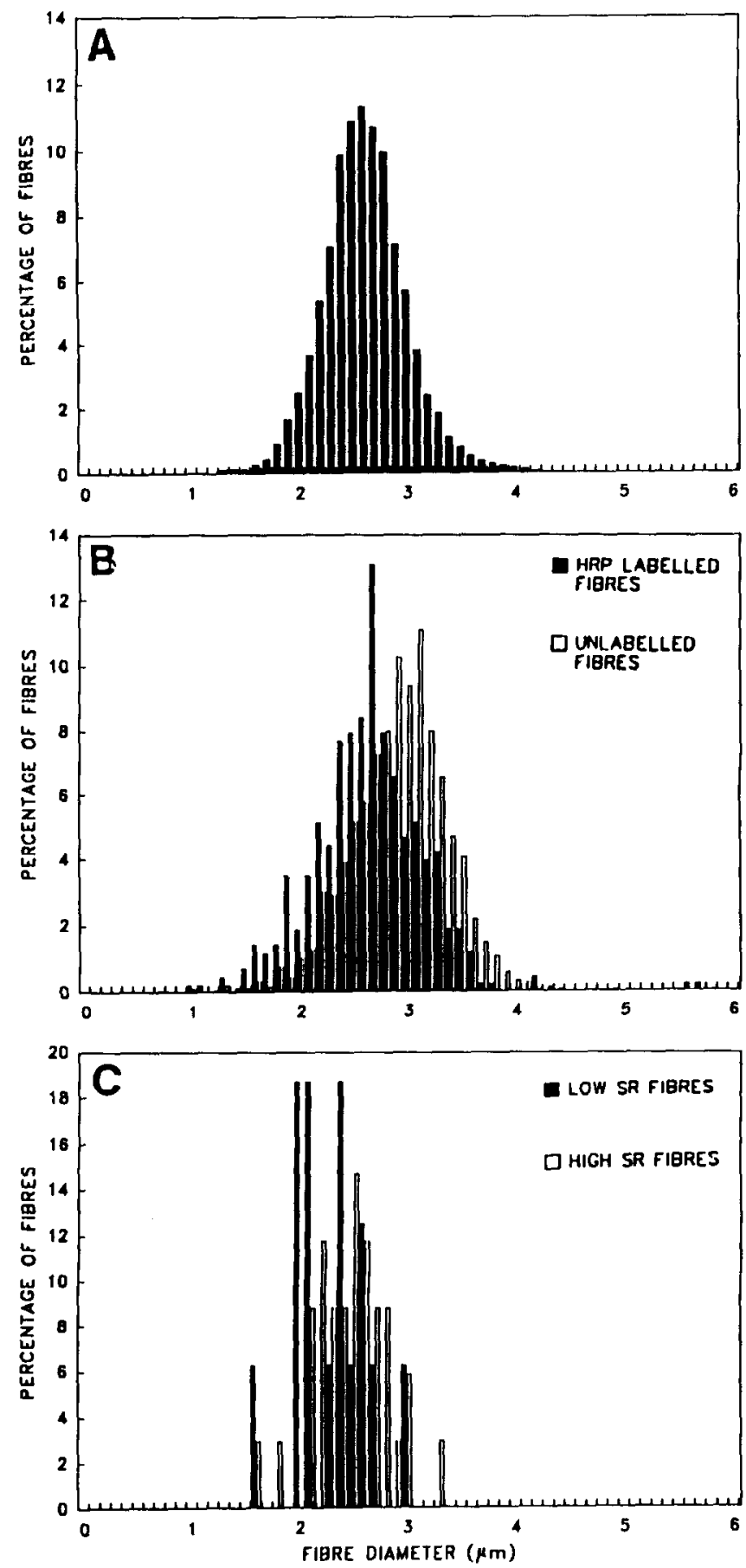

Fig. 4. Distribution of the internal diameters of auditory nerve fibres. (A), toluidine blue stained fibres from the basal half of the cochlea. Mean internal diameter $2.65 \mu \mathrm{m}$ (S.E.M. $=0.004 \mu \mathrm{m}, N=14173$ ). (B), Comparison of fibres labelled with HRP bulk injection into the spiral ganglion and adjacent unlabelled fibres. Mean diameter of HRP labelled fibres $2.70 \mu \mathrm{m}$ (S.E.M. $=0.03 \mu \mathrm{m}, N=428$ ), unlabelled fibres $2.97 \mu \mathrm{m}$ (S.E.M. $=0.01 \mu \mathrm{m}, N=1839$ ), this difference was significant $(P<0.01 ; t$-test). (C), comparison of low SR fibres (SR < 18) and high SR fibres (SR > 18) after intracellular application of HRP, measured $360 \mu \mathrm{m}$ central to Scarpa's ganglion for all 50 successfully labelled fibres. Mean for low SR fibres $2.25 \mu \mathrm{m}$ (S.E.M. $=0.08 \mu \mathrm{m}, N=16$ ), high SR fibres $2.37 \mu \mathrm{m}$ (S.E.M. $=0.06$ $\mu \mathrm{m}, N=34$ ), there was no significant difference.
TABLE I

Fibre diameter of HRP lahelled fihres and neighbouring unlabelled fibres

\begin{tabular}{lll}
\hline Animal & $\begin{array}{l}\text { Unlabelled fibres } \\
\text { mean diameter } \\
\text { and S.E.M. }(\mu \mathrm{m})\end{array}$ & $\begin{array}{l}\text { HRP labelled fibres } \\
\text { mean diameter } \\
\text { and S.E.M. }(\mu \mathrm{m})\end{array}$ \\
\hline $90 \mathrm{a}$ & $3.016 \pm 0.016$ & $2.634 \pm 0.041$ \\
$91 \mathrm{a}$ & $2.934 \pm 0.014$ & $2.732 \pm 0.032$ \\
\hline
\end{tabular}

$\mu \mathrm{m}$ (S.E.M. $=0.03 \mu \mathrm{m}, N=286$ ). This difference was not significant $(t$-test, $P>0.05)$.

\section{Diameter of low and high SR fibres}

For this study physiological data were only collected for cells from the high frequency region with CFs (most sensitive frequencies) ranging from $15-24 \mathrm{kHz}$. Single physiologically characterized fibres were grouped on the basis of their SR. Fibres with SR $<18$ spikes per second (range 0.0-4.8 spikes per second) were categorised as low SR and those with SR $>18$ spikes per second (range 21.2-107.6 spikes per second) as high SR. In a single animal only fibres belonging to one category were labelled by intracellular microelectrode injection of HRP. The typical appearance of such HRP injected fibres is shown in Figs. 2C and 2D.

In no case were more labelled fibres recovered than the number injected. The overall recovery rate for labelled fibres was $64 \%$. However, there was a marked difference in recovery rate for low SR (16 out of 37 attempts) and high SR (34 out of 41 attempts) fibres. For this part of the study we biased sampling towards low SRs. This was necessary because they were less frequently encountered and less successfully labelled. Thus we cannot determine the SR distribution from this data base. The average threshold at $\mathrm{CF}$ for the fibres that we tried to label was $26.6 \mathrm{~dB}$ SPL in the high SR group and $38.3 \mathrm{~dB}$ 'SPL in the low SR group. The higher thresholds in the low $\mathrm{SR}$ as compared to the high SR group are not due to a poor physiological condition of the cochlea, but rather reflect a physiological difference between the SR groups (Liberman, 1978; Schmiedt, 1989; Winter et al., 1990).

Fig. 4C shows the diameters of intracellularly labelled fibres which were determined $360 \mu \mathrm{m}$ central to Scarpa's ganglion. The range of diameters for low SR fibres was $1.51-3.06 \mu \mathrm{m}$ and for high SR fibres was 1.50-3.29 $\mu \mathrm{m}$. The mean diameter for low SR fibres (2.250 $\mu \mathrm{m}$, S.E.M. $=0.08 \mu \mathrm{m}, N=16)$ was smaller than that for high SR fibres $(2.367 \mu \mathrm{m}$, S.E.M. $=0.06$ $\mu \mathrm{m}, N=34$ ), but there was almost complete overlap and the difference was not significant ( $P>0.20, t$-test). The mean diameters of both low and high SR fibres were smaller than that of the bulk injected fibres. 


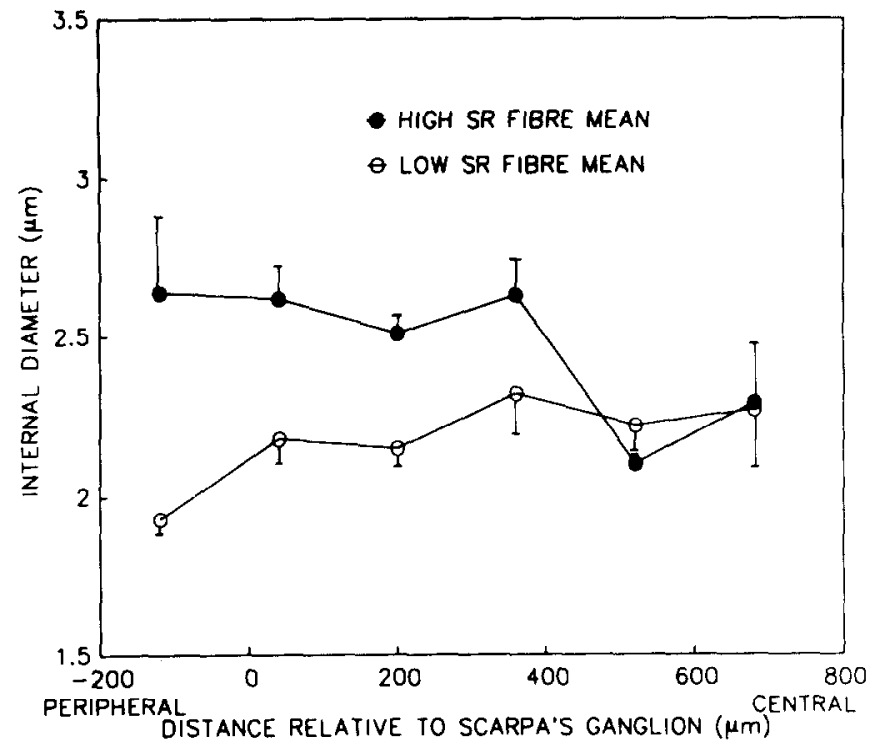

Fig. 5. Variation in mean diameter along the auditory nerve for selected low SR fibres $(N=7)$ and high SR tibres $(N=7)$. Io the left is peripheral to Scarpa's ganglion, to the right sections are close to the cochlear nucleus. The difference between the means was significant for the three most peripheral positions $(P<0.02 ; t$-test $)$.

\section{Fibre diameter along the auditory nerve}

To determine whether diameter varied along the nerve, a selected sample of 14 HRP-labelled fibres filled by intracellular injection were followed for approximately $800 \mu \mathrm{m}$ within the auditory nerve. In order to reduce the effort involved in this task we processed only two animals of the high SR as well as two animals of the low SR group for this part of the study. The criterion for selecting these specimens was that each contained several intracellularly labelled fibres (3-4) that could be uncquivocally recognized in subsequent sections according to their position in the nerve. The diameter of each of the fibres was determined at six positions approximately $160 \mu \mathrm{m}$ apart. The most peripheral position was just peripheral to Scarpa's ganglion and the most central position was near the cochlear nucleus.

In Fig. 5 the variation in mean diameter along the nerve for low and high SR fibres is shown. The mean diameter of high SR fibres $(N=7)$ was on average greater than that of low SR fibres $(N=7)$ for the four most peripheral positions. The difference in diamcter was significant for the three most peripheral positions $(P<0.02, t$-test $)$, while it was not statistically significant for the three more central positions. The difference between the means was greatest at the most peripheral position and progressively decreased along the nerve.

\section{Variation in fibre shape}

In a single cross section we found some difference in the shape (Fig. 2) of fibres and this same type of variation applied to individual fibres followed over some distance along the nerve. Shape factor measurements greater than 0 (associated with profiles deviating from circularity) were of concern because we calculated diameters from area measurements assuming circularity of the axonal profile. We found a significant correlation of fibre diameter and shape factor for a large sample of cells from a toluidine blue stained section $(\mathrm{r}=0.30, N=675, P<0.001)$. We also compared shape factor and diameter for fibres followed over $800 \mu \mathrm{m}$ in one animal. The correlation coefficient for this sample was similar $(r=0.32, N=30,0.05<P$ $<0.10$ ), but the significance level was, due to the smaller data base, decreased. Thus, the variation in shape factor explains only approximately $10 \%$ of the variation in diameter.

\section{Discussion}

\section{Distribution of fibre diameter}

As in human material (Spoendlin and Schrott, 1989), we could not identify unmyelinated profiles using light microscopy. Thus we exclusively analyzed fibres from Type I ganglion cells. The categorization of Type I auditory nerve fibres on the basis of physiological and morphological properties gave rise to the possibility of discrete populations within the Type I fibres. However the distribution of fibre diameter appears unimodal for a number of species. Gacek and Rasmussen (1961) obtained such distributions for fibre diameter in the guinea pig, cat and monkey. This finding has been confirmed for the cat (Arnesen and Osen, 1978) and guinea pig (Friede, 1984; this study) and was also reported for humans (Spoendlin and Schrott, 1989). The unimodal diameter distribution seems to be an universal feature across a variety of mammalian species.

\section{Processing affects fibre diameter measurements}

We clearly demonstrate that processing affects the results of diameter measurements (Figs. 3 and 4 ). The diameter of the unlabelled fibre population (Fig. 4B) was significantly larger than the average values obtained from toluoidine blue stained material (Fig. 4A) and HRP filled fibres (Fig. 4B). There are several possible reasons to explain differences in fibre diameter caused by different processing procedures. For example in the bulk HRP injections the peroxidase might preferentially be taken up by a subpopulation of thinner fibres, although thin and less active fibres are usually harder to label by HRP (Mesulam, 1982). A spatial organization of the cochlear ganglion in the cat with respect to SR distribution has recently been demonstrated (Leake and Snyder, 1989; Kawase and Liberman, 1992). Leake and Snyder (1989) used small focal injections $(0.05$ to $0.1 \mu \mathrm{l})$ of an HRP solution 
containing poly-L-ornithine to restrict the size of the injection site in the cochlear ganglion. We can not exclude the possibility that the comparatively small bulk injections of 0.1-0.2 $\mu$ l HRP solution we used might have reached only a restricted area of the ganglion that contains preferentially low SR fibres and could thus have labelled only a subpopulation of units. However, the average fibre diameters obtained from labelled fibres after bulk HRP injections $(2.7 \mu \mathrm{m})$ are very similar to the fibre diameters measured from toluidine blue stained material $(2.65 \mu \mathrm{m})$. In both situations the contrast between cytoplasm and the myelin sheat is rather high compared to the situation in unstained and unlabelled fibres. Thus the differing processing procedures may not have affected the fibre diameter per se, but rather influenced the measurements by changing the contrasts between cytoplasm and the myelin. In addition, toluidine blue, HRP and osmium stain and bind to different components of the cell and myelin sheath which in turn could affect the measurements. Clarification of this question would require a comparative ultrastructural study. Friede (1984) states that transmission electron microscopic and light microscopic measurements result in the same range of diameters, however he gives no quantitative comparison. In his sample fibres with diameters between 2.0 and $3.31 \mu \mathrm{m}$ comprise $70.6 \%$ of the whole population. We found that this diameter range forms $92.3 \%$ (toluidine blue), $80.2 \%$ (HRP bulk), 77.2\% (unlabelled and unstained) and 94\% (HRP intracellular) of the population studied.

Overall, these results show that comparisons between tissues processed using different methods are of limited value.

\section{The problem of oblique sectioning}

We found a significant positive correlation between shape factor and diameter for a large sample of axonal profiles in one cross section and a similar correlation coefficient for individual axonal profiles followed along the nerve. However, it explains only about $10 \%$ of the total variation in diameter that we found. It is hard to eliminate the possibility of oblique sectioning of some fibres running obliquely with respect to the plane of section. However there were a number of axonal profiles with shapes that would be difficult to obtain from oblique sectioning of a cylinder shaped axon, as can be seen in Fig. 2, including the 'boomerang' shape also observed by Karnes (1977). Indeed the low correlation coefficient between shape factor and diameter indicates that oblique sectioning is not a major source of the variation in diameter.

\section{Correlation of diameter and $S R$}

We found a significant difference between the average diameter of low and high SR fibres at the periph- ery near Scarpa's ganglion, but no difference near the cochlear nucleus (Fig. 5). The significant difference in diameter on the basis of SR for the peripheral positions measured (Fig. 5) implies there is some association between the two variables for the peripheral portion of the nerve. Fibres in our low SR category belong to the low and medium SR categories used by Liberman (1978) and those in our high SR category belong to Liberman's high SR category. We can therefore usefully compare our results with those obtained by Liberman and Oliver (1984) for the cat. They measured axonal diameter of HRP labelled fibres along the nerve, including the portion between the cell body and the cochlear nucleus. The mean diameters obtained by Liberman and Oliver for the peripheral half of this portion were $2.31 \mu \mathrm{m}$ (S.E.M. $=0.04 \mu \mathrm{m})$ for low and medium SR fibres and $2.54 \mu \mathrm{m}$ (S.E.M. $=0.06 \mu \mathrm{m}$ ) for high SR fibres. The portion of the nerve studied by Liberman and Oliver appears to correspond approximately to our three positions between $-120 \mu \mathrm{m}$ and $200 \mu \mathrm{m}$ relative to Scarpa's ganglion, representing the more peripheral portion of the auditory nerve beyond the Schwann-glia border. The present findings are similar to those of Liberman and Oliver (1984). Averaging fibre diameter for these positions (between $-120 \mu \mathrm{m}$ and $200 \mu \mathrm{m}$ relative to Scarpa's ganglion) we obtained a mean diameter of $2.10 \mu \mathrm{m}$ (S.E.M. $=0.04 \mu \mathrm{m}, N=7$ ) for low SR fibres, and $2.59 \mu \mathrm{m}$ (S.E.M. $=0.09 \mu \mathrm{m}$, $N=7$ ) for high SR fibres. As we did, Liberman and Oliver (1984) noted significant overlap in diameters between the groups so that a number of low SR fibres have diameters greater than high SR fibres (Fig. 4C). Thus the diameters of low and high SR auditory nerve fibres in the guinea pig are similar to those of the cat at the levels compared.

\section{The 'real' distribution of $S R s$ in the auditory nerve}

We hoped to obtain some information about the contribution of low and high SR units to the total SR distribution in the auditory nerve by comparing the diameter distributions of the different SR groups with the total diameter distribution. However, the poor correlation between SR and diameter made this approach untenable. Previously it has been suggested there might be a methodological bias against recording from low SR units duc to their suspected smaller diameter (Winter et al., 1990). Thus low SR units with rates below 18 spikes per second might comprise a higher proportion of fibres than the $20-25 \%$ found in, unintentionally biased, samples of guinea pig auditory nerve fibres (Manley and Robertson, 1976, own unpublished observations). However the distribution of diameters from fibres labelled with HRP using microelectrodes was similar to that obtained from bulk injected and toluidine blue stained fibres. In fact the mean diameters for low and high SR fibres identified after intracel- 
lular HRP injection were both slightly smaller than the mean diameter of bulk injected or unlabelled fibres. Nevertheless, like Winter et al. (1990) we also found that it is harder to record from low SR fibres and keep in contact with them for longer periods as compared to high SR cells. In addition we had a much lower recovery of labelled low SR fibres (43\%) than high SR fibres $(83 \%)$. It is known that labelling of small diameter fibres is compromised by the limited HRP transport (Brown et al., 1988; Mesulam, 1982). We thus can not exclude that we failed to label the really thin low SR fibres and succeeded only in a sample of low SR units with rather large diameters.

For cat data there exist also some discrepancies relating to the 'real' proportion of low and medium SR units derived from electrophysiological, morphological and single cell studies. In large samples of physiologically characterized units of cat auditory nerve fibres those with spontaneous discharge rates below approximately 18 spikes per second comprise approximately one third $(25-40 \%)$ of the total sample (Kim and Molnar, 1979; Liberman, 1978). In an ultrastructural investigation Liberman (1980) determined the complete afferent innervation of 4 inner hair cells from a cat with more than 100 afferents. In his quantitative study he found that about twice as many afferents contact the modiolar side of the hair cells as compared to the pillar side, where the dendrites are considerably larger. In a subsequent study Liberman (1982) demonstrated by intracellular labelling of physiologically characterized neurones that terminals of cat auditory nerve fibers are arranged systematically around the circumference of inner hair cells according to their SRs. Based on this SR-mapping around the base of inner hair cells (Fig. 2b in Liberman, 1982) he states that "terminal swellings contacting the side of the IHC facing the pillar or outer hair cells are exclusively those of high-SR units, while all the low- and medium-SR units contact the side of the IHC facing the modiolus'. In another study Liberman and Oliver (1982) report that in a sample of intracellularly labelled fibres 9 of the high SR units innervated the pillar side of inner hair cells while only 2 contacted the modiolar side. Of the low-SR units 13 contacted the modiolar side and no low-SR fibre innervated the pillar side of an inner hair ccll. Thus in the cat intraccllularly labellcd high-SR fibres almost exclusively $(>80 \%)$ innervate the pillar side of the inner hair cell. Combining the result of these studies (Liberman, 1980; 1982; Liberman and Oliver, 1984), one would predict that fibres with spontaneous discharge rates below 18 spikes per second comprise $50 \%$ or more of all auditory afferents. This is in contrast to the electrophysiologically determined proportion of $25-40 \%$ of low and medium SR fibres in the total sample (Liberman, 1978; Kim and Molnar, 1979). This discrepancy might be explained by the presence of low-SR units with very high thresholds, however, even using an electric search stimulus Liberman (1978) found that low- and medium-SR units formed only $40 \%$ of the total population Thus the question of the 'real' representation of different SRs with their differing rate-intensity functions (Winter et al., 1990) in the mammalian auditory nerve is not finally decided despite its importance for the understanding and modelling of the intensity discrimination performance of mammals.

\section{Acknowledgements}

This work was supported by the National Health and Medical Research Council of Australia. We wish to thank Dr. D. Robertson for his advice and support throughout the project, Barbara Gleich for patiently carrying out the sectioning and Dr. S. Dyson for assistance with the MD20 Image Analysis System.

\section{References}

Alder, V.A. and B.M. Johnstone (1978) A new approach to the guinea pig auditory nerve. J. Acoust. Soc. Am., 64, 684-687.

Alving, B.M. and W.M. Cowan (1971) Some quantitative observations on the cochlear division of the eighth nerve in the squirrel monkey (Saimiri sciureus). Brain Res. 25, 229-239.

Anniko, M. and A.R. Arnesen (1988) Cochlear nerve topography and fiber spectrum in the pigmented mouse. Arch. Otorhinolaryngol., $245,155-159$

Arnesen, A.R. and K.K. Osen (1978) The cochlear nerve in the cat: topography, cochleotopy and fiber spectrum. J. Comp. Neurol., 178, 661-678.

Brown, M.C. (1987) Morphology of labeled afferent fibers in the guinea pig cochlea. J. Comp. Neurol., 260, 591-604.

Brown, M.C., Berglund, A.M., Kiang, N.Y.S. and Kyugo, D.K. (1988) Central trajectories of Type II spiral ganglion neurons. J. Comp. Neurol., 278, 581-590.

Friede, R.L. (1984) Cochlear axon calibres are adjusted to characteristic frequencies. J. Neurol. Sci., 66, 193-200.

Gacek, R.R. and G.L. Rasmussen (1961) Fiber analysis of the statoacoustic nerve of guinea pig, cat and monkey. Anat. Rec., $139,455-463$.

Karnes, J., R. Robb, P.C. O'Brian, E.H. Lambert and P.J. Dyck (1977) Computerized image recognition for morphometry of nerve attribute of shape of sampled transverse sections of myelinated fibers which best estimates their average diameter. J. Neurol. Sci., 34, 43-51.

Kawase, T. and Liberman, M.C. (1992) Spatial organization of the auditory nerve according to spontaneous discharge rate. J. Comp. Neurol., 319, 312-318.

Kiang, N.Y.S., J.M. Rho, C.C. Northrop, M.C. Liberman and D.K. Ryugo (1982) Hair-cell innervation by spiral ganglion cells in adult cats. Science, $217,175-177$.

Leake, P.A. and Snyder, R.L. (1989) Topographic organization of the central projections of the spiral ganglion in cats, J. Comp. Neurol., 281, 612-629.

Kim, D.O. and Molnar, C.E. (1979) A population study of cochlear nerve fibers: Comparison of spatial distributions of average-rate and phase-locking measures of responses to single tones. J. Neurophysiol., 42, 16-30 
Liberman, M.C. (1978) Auditory-nerve response from cats raised in a low-noise chamber. J. Acoust. Soc. Am., 63, 442-455.

Liberman, M.C. (1980) Morphological differences among radial afferent fibers in the cat cochlea: An electron-microscopic study of serial sections. Hear. Res., 3, 45-63.

Liberman, M.C. (1982) Single-neuron labeling in the cat auditory nerve. Science, 216, 1239-1241.

Liberman, M.C. (1988) Physiology of cochlear efferent and afferent neurons: Direct comparisons in the same animal. Hear. Res., 34, 179-192.

Liberman, M.C. and M.E. Oliver (1984) Morphometry of intracellularly labeled neurons of the auditory nerve: correlations with functional properties. J. Comp. Neurol., 223, 163-176.

Manley, G.A. and Robertson, D. (1976) Analysis of spontaneous activity of auditory neurones in the spiral ganglion of the guineapig cochlea. J. Physiol., 258, 323-336.

Mesulam, M.-M. (1982) Principles of horseradish peroxidase neurohistochemistry and their applications for tracing neural pathways-axonal transport, enzyme histochemistry and light microscopic analysis. In: M.-M. Mesulam (Ed.) Tracing Neural Connections with Horseradish Peroxidase, Wiley \& Sons, Chichester, pp 1-151.

Rhode, W.S. and P.H. Smith (1985) Characteristics of tone-pip response patterns in relationship to spontaneous rate in cat auditory nerve fibers. Hear. Res., 18, 159-168.

Rohertson, D. (1984) Horseradish peroxidase injection of physically characterised afferent and efferent neurones in the guinea pig spiral ganglion. Hear. Res., 15, 113-121.
Robertson, D. and G.A. Manley (1974) Manipulation of frequency analysis in the cochlear ganglion of the guinea pig. J. Comp. Physiol., 91, 363-375.

Sachs, M.B. and Abbas, P.J. (1974) Rate versus level functions for auditory nerve fibres in cats: tone burst Stimuli. J. Acoust. Soc. Am. 56, 1835-1847.

Sachs, M.B., R.L. Winslow and B.H.A. Sokolowski (1989) A computational model for rate-level functions from cat auditory-nerve fibres. Hear. Res., 41, 61-70.

Schmiedt, R.A. (1989) Spontaneous trates, thresholds and tuning of auditory-nerve fibers in the gerbil: Comparison to cat data. Hear. Res., 42, 23-36.

Spoendlin, H. (1971) Degeneration behaviour of the cochlear nerve. Arch. Ohr. Nas. Kehlk. Heilk., 200, 275-291.

Spoendlin, H. (1972) Innervation densities of the cochlea. Acta Otolaryngol., $73,235-248$.

Spoendlin, H (1979) Neural connections of the outer haircell system. Acta Otolaryngol., 87, 381-387.

Spoendlin, H. and A. Schrott (1989) Analysis of the human auditory nerve. Hear. Res., 43, 25-38.

Winter, I. $\Lambda$., D. Robertson and G.K. Yates (1990) Diversity of characteristic frequency rate-intensity functions in guinea pig auditory nerve fibres. Hear. Res., 45, 191-202.

Yates, G.K., I.A. Winter and D. Robertson (1990) Basilar membrane nonlinearity determines auditory nerve rate-intensity functions and cochlear dynamic range. Hear. Res., 45, 203-220. 